\title{
P-Adic Feynman and String Amplitudes
}

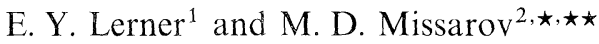 \\ 1 Kazan State University, Kazan, Kazan, USSR \\ ${ }^{2}$ Centre de Physique Théorique, CNRS-Luminy, Marseille, France
}

\begin{abstract}
We derive an explicit representation for $p$-adic Feynman and Koba-Nielsen amplitudes and we briefly outline the connection between the scalar models of $p$-adic quantum field theory and Dyson's hierarchical models.
\end{abstract}

\section{Introduction}

As we have shown previously (see [1], submitted to "Theoretical and Mathematical Physics" in May 1987), the scalar models of the field theory over the p-adic field $Q_{p}$ are the natural continuous analogs of Dyson's hierarchical models (see [2-5]). More precisely, the discretization of the field theory over $Q_{p}$ on the hierarchical lattice of $p$-adic numbers with zero integer part is a model of Dyson's type. The traditional methods of quantum field theory such as Feynman diagrams, renormalization theory and Wilson's renormalization group have analogs in the $p$-adic case. The main results of [1] are briefly outlined in Sect. 2 .

On the other hand, there has been recently some interest on the possibility of a $p$-adic formulation of string theory (see [5-12]).

All this explains our interest in the Feynman amplitudes over the $p$-adic field. The remarkable feature of $p$-adic models is the exact representation of Feynman and string scattering amplitudes as a sum of elementary functions. Namely, let us consider a general Feynman amplitude over $Q_{p}$ in coordinate representation,

$$
F\left(x_{v} ; v \in V_{\mathrm{ext}}\right)=\int \prod_{v, v^{\prime} \in V_{\mathrm{ext}} \cup V_{\mathrm{int}}}\left\|x_{v}-x_{v^{\prime}}\right\|_{p}^{a\left(v, v^{\prime}\right)} \prod_{v \in V_{\mathrm{int}}} d x_{v}
$$

where the integral taken over $Q_{p}^{\left|V_{\text {int }}\right|}, p$ is a fixed prime number, $Q_{p}$ is a $p$-adic field, $\|\cdot\|_{p}$ is a $p$-adic norm (further on, the sign $p$ will be omitted), $V_{\text {ext }}\left(V_{\text {int }}\right)$ is a set of external (internal) vertices, $a\left(v, v^{\prime}\right) \in \mathbb{C}$ for each pair $v \in V_{\text {ext }} \cup V_{\text {int }}, v^{\prime} \in V_{\text {ext }} \cup V_{\text {int }}$ (we identify a pair $\left(v, v^{\prime}\right)$ with $\left.\left(v^{\prime}, v\right)\right), d x$ is a Haar measure on $Q_{p}$, normalized such

« Permanent address: Department of Applied Mathematics, Kazan State University, Kazan, USSR

* This work was supported in part by the French Government 
that

$$
\int_{|x| \leq 1} d x=1
$$

Every given vector of external variables $x=\left(x_{v} ; v \in V_{\text {ext }}\right) \in Q_{p}^{\left|V_{\text {ext }}\right|}$ generates on $V_{\text {ext }}$ a hierarchy $A_{x}$. We recall (see, for example $[4,12]$ ) that a hierarchy $A$ on a finite set $V$ is a family of subsets of $V$, such that $V \in A,\{v\} \in A$ for every $v \in V$, and for each pair $V^{\prime} \in A^{\prime}, V^{\prime \prime} \in A$ either $V^{\prime} \cap V^{\prime \prime}=\varnothing$ or $V^{\prime} \subset V^{\prime \prime}$, or $V^{\prime \prime} \subset V^{\prime}$. For every $V^{\prime} \in A$ we denote by $\tau\left(V^{\prime}\right)$ the minimal set in $A$, which contains $V^{\prime}$, but does not coincide with $V^{\prime}$ (we assume $V^{\prime} \neq V$ ). In the following we shall consider only hierarchies such as

$$
1<\left|K\left(V^{\prime}\right)\right| \leqq p, \quad V^{\prime} \in A^{\prime}
$$

whiere

$$
\begin{aligned}
K\left(V^{\prime}\right) & =\left\{V^{\prime \prime} \in A \mid \tau\left(V^{\prime \prime}\right)=V^{\prime}\right\}, \\
A^{\prime} & =\left\{V^{\prime} \in A:\left|V^{\prime}\right|>1\right\} .
\end{aligned}
$$

In our case, the hierarchy $A_{x}$ on $V_{\text {ext }}$ is defined as

$$
A_{x}=\left\{V \subset V_{\text {ext }}: \max _{\substack{v \in V \\ v^{\prime} \in V}}\left\|x_{v}-x_{v^{\prime}}\right\|<\min _{\substack{v \in V \\ v^{\prime} \in V_{\mathrm{ext}} \backslash V}}\left\|x_{v}-x_{v^{\prime}}\right\|\right\} .
$$

Note, that for every $A$ on $V_{\text {ext }}$ there exists $x \in Q_{p}^{\left|V_{\text {ext }}\right|}$ such that $A_{x}=A$.

Let

$$
F_{A}(x)= \begin{cases}F(x), & \text { if } A_{x}=A \\ 0, & \text { otherwise }\end{cases}
$$

Then

$$
F(x)=\sum_{A} F_{A}(x)
$$

where the sum goes over all hierarchies on $V_{\text {ext }}$.

Let $A_{x}=A$. The main result of Sect. 3 is the following:

$$
F_{A}(x)=\sum_{I(A)} \prod_{V \in A^{\prime}} C(V, I) \max _{\substack{v \in V \\ v^{\prime} \in V}}\left\|x_{v}-x_{v^{\prime}}\right\|^{\lambda(V, I)}
$$

where the sum taken over all partitions of $V_{\text {int }}$, indexed by the elements of $A^{\prime}$ :

$$
\begin{gathered}
I(A)=\left\{I(V), V \in A^{\prime}\right\}, \quad I\left(V^{\prime}\right) \cap I\left(V^{\prime \prime}\right)=\varnothing, \quad \text { if } \quad V^{\prime} \neq V^{\prime \prime}, \quad\left(\bigcup_{V \in A^{\prime}} I\left(V^{\prime}\right)\right)=V_{\mathrm{int}}, \\
\lambda(V, I)=a(V(I))-\sum_{V^{\prime} \in K(V)} a\left(V^{\prime}(I)\right)+|I(V)|, \\
V(I)=\left(\bigcup_{V^{\prime} \subseteq V} I\left(V^{\prime}\right)\right) \cup V \\
C(V, I)=\int \prod_{v, v^{\prime} \in I(V)}\left\|y_{v}-y_{v^{\prime}}\right\|^{a\left(v, v^{\prime}\right)} \prod_{v \in I(V)}\left(\prod_{V^{\prime} \in K^{\prime}(V)}\left\|y_{v}-\alpha_{V^{\prime}}\right\|^{a\left(v, V^{\prime}(I)\right)}\right) d y
\end{gathered}
$$


Integral (1.13) taken over $Q_{p}^{\mid I(V) !},\left\{\alpha_{V^{\prime}}, V^{\prime} \in K(V)\right\}$ is an arbitrary set of $p$-adic numbers, such that $\left\|\alpha_{V^{\prime}}-\alpha_{V^{\prime \prime}}\right\|=1$, if $V^{\prime} \neq V^{\prime \prime}$ (coefficient $C(V, I)$ does not depend on the choice of $\alpha=\left\{\alpha_{V}, V^{\prime} \in K(V)\right\}$. Here we use the notations

$$
\begin{gathered}
a\left(W, W^{\prime}\right)=\sum_{v \in W, v^{\prime} \in W^{\prime}} a\left(v, v^{\prime}\right), \quad W, W^{\prime} \subset V_{\mathrm{ext}} \cup V_{\mathrm{int}}, \\
a(W)=a(W, W) .
\end{gathered}
$$

We see that the integral representation for $C(V, I)$ is a generalization of the integral representation of string scattering amplitude in the Koba-Nielsen form.

Let

$$
F_{1}=\int \prod_{v, v^{\prime} \in V}\left\|y_{v}-y_{v^{\prime}}\right\|^{a\left(v, v^{\prime}\right)} \prod_{v \in V} \prod_{i=0}^{k}\left\|y_{v}-\alpha_{i}\right\|^{a(v, i)} d y_{v}
$$

where $0 \leqq k<p, \alpha=\left\{\alpha_{i}, i=0, \ldots, k\right\}$ is an arbitrary set of $p$-adic numbers, such that $\left\|\alpha_{i}-\alpha_{j}\right\|=1$, if $i \neq j, V$ is a finite set, $a\left(v, v^{\prime}\right) \in C$ for each pair $v \in V, v^{\prime} \in V$ (we identify $\left(v, v^{\prime}\right)$ with $\left.\left(v^{\prime}, v\right)\right)$. In Sect. 4 we prove that the calculation of this integral can be reduced to that of the following one:

$$
F_{2}=\int_{\substack{\left\{y_{v} \mid<1 \\ v \in V \backslash\left\{v_{0}\right\}\right.}} \prod_{v, v^{\prime} \in V}\left\|y_{v}-y_{v^{\prime}}\right\|^{a\left(v, v^{\prime}\right)} \prod_{v \in V \backslash\left\{v_{0}\right\}} d y_{v}
$$

where $y_{v_{0}}=0$. We shall prove that

$$
F_{2}\left(a_{V}\right)=p^{-|V|+1} \sum_{A} \prod_{V^{\prime} \in A^{\prime}} \frac{1}{p^{a\left(V^{\prime}\right)+\left|V^{\prime}\right|-1}-1} \cdot \frac{(p-1) !}{\left(p-\left|K\left(V^{\prime}\right)\right|\right) !},
$$

the sum taken over all hierarchies $A$ on $V$.

\section{P-Adic Scalar Models and Dyson's Hierarchical Models}

Let a generalized random field $P(\phi)$ on the $Q_{p}$ be given, i.e. a system of probability distributions $P\left\{\left(\varphi, f_{1}\right), \ldots,\left(\varphi, f_{m}\right)\right\}$ with the usual conditions of accordance. Here $f_{i}=f_{i}(x), i=1, \ldots, m$ are arbitrary test functions in the space $S\left(Q_{p}\right) . S\left(Q_{p}\right)$ is a space of locally piece-wise finite complex-valued functions on $Q_{p}$ (see [15]).

We define the scaling operator

$$
R_{\lambda}^{a} P(\varphi)=P\left(\|\lambda\|^{1-(a / 2)} \varphi_{\lambda}\right)
$$

where $a$ is a real number, $1 \leqq a \leqq 2, \lambda \in Q_{p}$,

$$
\left(\varphi_{\lambda}, f\right)=(\varphi(\lambda x), f(x))
$$

and $P\left(\|\lambda\|^{1-(a / 2)} \varphi_{\lambda}\right)$ is a generalized random field with probability distributions

$$
P\left\{\left(\|\lambda\|^{1-(a / 2)} \varphi_{\lambda}, f_{1}\right), \ldots,\left(\|\lambda\|^{1-(a / 2)} \varphi_{\lambda}, f_{m}\right)\right\} .
$$

A generalized random field is scaling invariant if

$$
R_{\lambda}^{a} P(\varphi)=P(\varphi)
$$

for all $\lambda \in Q_{p}$. A generalized random field is called translation invariant if $P(\varphi(x))=P(\varphi(x+a))$ for any $a \in Q_{p}$. 
It is easy to see that a generalized gaussian random field with zero mean and binary correlation function

$$
\left\langle\varphi\left(x_{1}\right), \varphi\left(x_{2}\right)\right\rangle=\mathrm{const}\left\|x_{1}-x_{2}\right\|^{a-2}
$$

is a scaling and translation invariant random field.

By $\mathscr{D}_{p}$ we denote the set of all $p$-adic numbers with zero integer part. If

$$
x=\sum_{i=n}^{\infty} a_{i} p^{i}, \quad 0 \leqq a_{i}<p-1
$$

is a $p$-adic number, then $\{x\}$ denotes its fraction part

$$
\begin{aligned}
& \{x\}=\sum_{i=n}^{-1} a_{i} p^{i}, \\
& \mathscr{D}_{p}=\left\{x \in Q_{p}: x=\{x\}\right\} .
\end{aligned}
$$

$\mathscr{D}_{p}$ has a natural hierarchical structure, which consists from all sets of the type

$$
V_{i}^{n}=\left\{x \in \mathscr{D}_{p}:\left\|p^{n} x-i\right\| \leqq 1\right\}, \quad i \in \mathscr{D}_{p}, \quad n=0,1,2, \ldots
$$

The discretization of a generalized random field $\varphi$ on $\mathscr{D}_{p}$ is defined as a random field $\xi$ on $\mathscr{D}_{p}$ such that

$$
\xi=\left\{\xi_{j}=\left(\varphi, \chi_{j}\right), j \in \mathscr{D}_{p}\right\},
$$

where $\chi_{j}(x)=\chi(x-j), \quad \chi(x)$ is the characteristic function of the ball $Z_{p}=$ $\left\{x \in Q_{p}:\|x\| \leqq 1\right\}$. The following operations on a random field $\xi=\left\{\xi_{j}, j \in \mathscr{D}_{p}\right\}$ are defined by the formulae

$$
\begin{gathered}
r_{\lambda}^{a}: \xi_{j} \rightarrow \xi_{j}^{\prime}=\|\lambda\|^{-(a / 2)} \sum_{\substack{i:\{t,-1\}=1, j \in \mathscr{D}_{p} \\
t_{i}}} \xi_{i}, \quad\|\lambda\| \geqq 1, \\
\xi_{j} \rightarrow \xi_{\{1+i\}}, \quad i \in \mathscr{D}_{p} .
\end{gathered}
$$

If a generalized random field $\varphi$ is a translation and scaling invariant, then its discretization is invariant relative to actions $t_{i}, i \in \mathscr{D}_{p}$ and $r_{\lambda}^{a},\|\lambda\|>1$. The gaussian scaling and translation invariant random field on $Q_{p}$ also may be defined by the hamiltonian

$$
H_{0}=\frac{1}{2} \int\|x-y\|^{-a} \varphi(x) \varphi(y) d x d y .
$$

One can show that the discretization of the random field with the hamiltonian (2.11) is a gaussian random field on $\mathscr{D}_{p}$ with the hamiltonian

where

$$
\tilde{H}_{0}=\frac{1}{2} \sum_{i, j \in \mathscr{Z}_{p}} d(i, j) \xi_{i} \xi_{j},
$$

$$
d(i, j)=\left\{\begin{array}{ll}
\|i-j\|^{-a}, & \text { if } i \neq j \\
\frac{p^{-a}-p^{1-a}}{1-p^{1-a}}, & \text { otherwise }
\end{array} .\right.
$$

Note that the hamiltonian $\widetilde{H}_{0}$ is the hamiltonian of the gaussian Dyson hierarchical model. 
Further we shall have to deal with the hamiltonians in momentum representation. A hamiltonian in the ball $\Omega=\left\{k \in Q_{p}\right.$ : $\left.\|k\| \leqq R\right\}$ is an expression of the form

$$
H(\sigma)=\sum_{m=1}^{n} \int_{\Omega^{m}} h_{m}\left(k_{1}, \ldots, k_{m}\right) \delta\left(k_{1}+\cdots+k_{m}\right) \prod_{i=0}^{k} \sigma\left(k_{i}\right) d k_{i} .
$$

A formal hamiltonian is a formal series in $\varepsilon$

$$
H=H_{0}+\varepsilon H_{1}+\varepsilon^{2} H_{2}+\ldots,
$$

whose coefficients are finite-particle hamiltonians of the type (2.14). In what follows the coefficient $H_{0}$ will be fixed:

$$
H_{0}=\frac{1}{2} \int_{\Omega}\|k\|^{a-1}|\sigma(k)|^{2} d k
$$

Wilson's renormalization transformation, as in a real case, is defined by the formula

$$
R_{\Omega, \lambda}^{a} H=\ln \left\langle\exp \left(R_{\lambda,}^{a} H\left(\sigma_{0}+\sigma_{1}\right)\right)\right\rangle_{\mu\left(d \sigma_{1}\right)}
$$

where $\left(R_{\lambda}^{a} H\right)(\sigma)=H\left(\|\lambda\|^{-a / 2} \sigma\left(\lambda^{-1}\right)\right)$ is a hamiltonian of the random field in the ball $\lambda \Omega, \sigma(k)$ is a configuration in the ball $\lambda \Omega, \sigma(k)=\sigma_{0}(k)+\sigma_{1}(k), \sigma_{0}(k)=\chi_{\Omega}(k) \sigma(k)$, $\sigma_{1}(k)=\left(\chi_{i \Omega}(k)-\chi_{\Omega}(k)\right) \sigma(k), \chi_{\Omega}(k)$ is a characteristic function of the ball $\Omega$, and the average taken with respect to the gaussian measure $\mu\left(d \sigma_{1}\right)$ with zero mean and binary correlation function

$$
\left\langle\sigma_{1}(k) \sigma_{1}\left(k^{\prime}\right)\right\rangle=\delta\left(k+k^{\prime}\right)\left(\chi_{\lambda \Omega}(k)-\chi_{\Omega}(k)\right)\|k\|^{1-a} .
$$

The branch of gaussian fixed points of Wilson's renormalization group is determined by the hamiltonian $H_{0}=H_{0}(a)$. An investigation of the spectrum of the differential of the renormalization group on this branch shows that $a_{0}=\frac{3}{2}$ is the bifurcation point. One can try to construct a new branch of non-gaussian fixed points as power series in the deviation of the parameter $a$ from the bifurcation value $a_{0}$. As in the real case, (see [16]), we seek a solution in the class of projection hamiltonians, in the form

$$
H=H(\sigma, \varepsilon)=\ln \left\langle\exp \left\{u(\varepsilon) \varphi^{4}\left(\sigma_{0}+\sigma_{1}\right)\right\}\right\rangle_{\mu\left(d \sigma_{1}\right)},
$$

where

$$
\varphi^{4}(\sigma)=\int \delta\left(k_{1}+\cdots+k_{4}\right) \prod_{i=1}^{4} \sigma\left(k_{i}\right) d k_{i},
$$

$\sigma_{0}$ is a configuration in the ball $\Omega$, and the average taken with respect to the gaussian measure with binary correlation function

$$
\left\langle\sigma_{1}(k) \sigma\left(k^{\prime}\right)\right\rangle=\delta\left(k+k^{\prime}\right)\left(1-\chi_{\Omega}(k)\right)\|k\|^{1-a}=\delta\left(k+k^{\prime}\right)\left(1-\chi_{\Omega}(k)\right)\|k\|^{-(1 / 2)-\varepsilon} .
$$

The only quantity which is not defined here is $u(\varepsilon)$, which we assume to be a formal power series in $\varepsilon$ :

$$
u(\varepsilon)=\sum_{i=1}^{\infty} u_{j} \varepsilon^{j}
$$

In the computation of a projection hamiltonian divergences appear. Namely, in 
$\varphi^{4}$ theory with the propagator

$$
\left(1-\chi_{\Omega}(k)\right)\|k\|^{-(1 / 2)-\varepsilon}
$$

the diagrams with two and four external lines have poles when $\varepsilon \rightarrow 0$. The theory of analytic renormalization analogous to the real case exists in the $p$-adic case. One can show that

$$
\begin{aligned}
& R_{\Omega, \lambda}^{a} \text { A.R. } \ln \left\langle\exp \left\{u(\varepsilon) \varphi^{4}\left(\sigma_{0}+\sigma_{1}\right)\right\}\right\rangle_{\mu\left(d \sigma_{1}\right)} \\
& \quad=\exp \left(\tau \beta(u) \frac{d}{d u}\right) \text { A.R. } \ln \left\langle\exp \left\{u(\varepsilon) \varphi^{4}\left(\sigma_{0}+\sigma_{1}\right)\right\}\right\rangle_{\mu\left(d \sigma_{1}\right)},
\end{aligned}
$$

where A.R. denotes the analytic renormalization with minimal subtractions, $\tau=2 \ln \|\lambda\|$,

$$
\beta(u)=\varepsilon u+\sum_{n=2}^{\infty} c_{n} u^{u},
$$

and the coefficients $c_{n}$ for $n \geqq 2$ do not depend on $\varepsilon$.

The renormalization projection hamiltonian A.R. $\ln \left\langle\exp \left(u(\varepsilon) \varphi_{4}\right)\right\rangle_{\mu\left(d \sigma_{1}\right)}$ is invariant under the action of the renormalization group, if

$$
\beta(u)=0 \text {. }
$$

This equation has two solutions in the formal power in $\varepsilon$. The solution $u=0$ corresponds to a gaussian fixed point. A nontrivial non-gaussian solution is obtained from the solution of

$$
\varepsilon+\sum_{n=2}^{\infty} c_{n} u^{n-1}=0
$$

It is easy to check that $c_{2} \neq 0$ and therefore Eq. (2.27) is indeed solvable. This solution is a $p$-adic field-theoretical description of the non-trivial solution in Dyson's hierarchical model, which was investigated in [2--5].

\section{Feynman Amplitudes in Coordinate Representation}

First of all we shall prove that $A_{x}$, defined by (1.6) with the function

$$
m_{x}(V)=\max _{\substack{v \in V \\ v^{\prime} \in V}}\left\|x_{v}-x_{v^{\prime}}\right\|
$$

on it, is an indexed hierarchy. We recall that an indexed hierarchy on $V$ is a pair $(A, m)$, where $A$ denotes a given hierarchy on $V$ and $m$ is a positive function on $A$, satisfying the following conditions:

1) $m\left(V^{\prime}\right)=0$ if and only if $\left.\mid V^{\prime}\right\}=1$,

2) if $V^{\prime} \subset V^{\prime \prime}$, then $m\left(V^{\prime}\right)<m\left(V^{\prime \prime}\right)$.

In addition to these conditions we shall consider the functions $m(V)$ for which

for every $V \in A$.

$$
m(V)=p^{n(V)}, \quad n(V) \in Z
$$


It suffices to show that if $V^{\prime} \in A_{x}, V^{\prime \prime} \in A_{x}$ and $V^{\prime} \cap V^{\prime \prime} \neq \varnothing$, then $V^{\prime} \subset V^{\prime \prime}$ or $V^{\prime \prime} \subset V^{\prime}$. In fact, let $x \in V^{\prime} \cap V^{\prime \prime}, y \in V^{\prime} \backslash V^{\prime \prime}, z \in V^{\prime \prime} \backslash V^{\prime}$. From the definition of $V^{\prime}$ $\|x-y\|<\|y-z\|$ follows and hence, from the ultrametricity of the $p$-adic norm $\|y-z\|=\|x-z\|$, but this contradicts the definition of $V^{\prime \prime}$.

It is easy to see that for every indexed hierarchy $(A, m)$ on $V_{\text {ext }}$ there exists $x=\left(x_{v}, v \in V_{\text {ext }}\right)$ such that $\left(A_{x}, m_{x}\right)=(A, m)$. To every indexed hierarchy $(A, m)$ corresponds a family of sets $\{\mathscr{D}(V, m), V \in A\}$, where

$$
\mathscr{D}(V, m)=\left\{y \in Q_{p}: m(V) \leqq\left\|y-x_{v}\right\|<m(\tau(V)), \forall v \in V\right\} .
$$

We put $m\left(\tau\left(V_{\text {ext }}\right)\right)=\infty$.

Let $\varphi: A \rightarrow V_{\text {ext }}$ be any function such that $\varphi(V) \in V$ for every $V \in A$.

Lemma 1. 1) If $V \in A, V^{\prime} \in A, V \subset V^{\prime}, V \neq V^{\prime}$ and $y \in \mathscr{D}(V, m), y \in \mathscr{D}\left(V^{\prime}, m\right)$. then

$$
\left\|y^{\prime}-y\right\|=\left\|y^{\prime}-x_{\varphi(V)}\right\| \text {. }
$$

2) If $V \cap V^{\prime}=\varnothing, V \in A, V^{\prime} \in A$ and $V^{\prime \prime}$ is a minimal set in $A$, containing $V \cup V^{\prime}$, $y \in \mathscr{D}(V, m), y^{\prime} \in \mathscr{D}\left(V^{\prime}, m\right)$, then

$$
\left\|y^{\prime}-y\right\|=m\left(V^{\prime \prime}\right)
$$

3) $\{\mathscr{D}(V, m) ; V \in A\}$ is a partition of $Q_{p}$.

Proof. Let $y \in \mathscr{D}(V, m), y^{\prime} \in \mathscr{D}\left(V^{\prime}, m\right), V \subset V^{\prime}, V \neq V^{\prime}$.

Then

$$
\left\|y-x_{\varphi(V)}\right\|<m(\tau(V)) \leqq m\left(V^{\prime}\right) \leqq\left\|y^{\prime}-x_{\varphi(V)}\right\|,
$$

and therefore $\left\|y-y^{\prime}\right\|=\left\|y^{\prime}-x_{\varphi(V)}\right\|$. If $V \cap V^{\prime}=\varnothing, y \in \mathscr{D}(V, m), y^{\prime} \in \mathscr{D}\left(V^{\prime}, m\right)$, then

and hence

$$
\begin{gathered}
\left\|y-x_{\varphi(V)}\right\|<m(\tau(V)) \leqq m\left(V^{\prime \prime}\right)=\left\|x_{\varphi(V)}-x_{\varphi\left(V^{\prime}\right)}\right\|, \\
\left\|y^{\prime}-x_{\varphi\left(V^{\prime}\right)}\right\|<m\left(\tau\left(V^{\prime}\right)\right) \leqq m\left(V^{\prime \prime}\right)
\end{gathered}
$$

$$
\left\|y^{\prime}-x_{\varphi(V)}\right\|=\left\|y-y^{\prime}\right\|=m\left(V^{\prime \prime}\right)
$$

Let $V \neq V^{\prime}$ and $y \in \mathscr{D}(V, m) \cap \mathscr{D}\left(V^{\prime}, m\right)$. If $V \subset V^{\prime}, V \neq V^{\prime}$, then

$$
m(V) \leqq\left\|y-x_{\varphi(V)}\right\|<m(\tau(V)) \leqq\left\|y-x_{\varphi(V)}\right\|,
$$

but this contradicts the first part of the lemma. If $V \cap V^{\prime \prime}=\varnothing$, then

$$
\left\|x_{\varphi(V)}-x_{\varphi\left(V^{\prime}\right)}\right\|<\max \left(\left\|y-x_{\varphi(V)}\right\|,\left\|y-x_{\varphi\left(V^{\prime}\right)}\right\|\right)<m\left(V^{\prime \prime}\right) .
$$

At last

$$
\bigcup_{V \in A} \mathscr{D}(V, m)=Q_{p}
$$

Lemma 1 is proved.

Everywhere below we have used the next notations: $a_{V}=\left(a\left(v, v^{\prime}\right)\right)_{v \in V}^{v^{\prime} \in V}$ is a matrix, $b_{V}=(b(v))_{v \in V}$ is a vector,

$$
a\left(V, V^{\prime}\right)=\sum_{v \in V, v^{\prime} \in V^{\prime}} a\left(v, v^{\prime}\right)
$$


$a(V)=a(V, V), a_{V}^{V^{\prime}}=\left(a\left(v, V^{\prime}\right)\right)_{v \in V}$ is a vector, $a\left(v, v^{\prime}\right) \in \mathbb{C}, b^{\prime}(v) \in \mathbb{C}$ for every $v \in V, v^{\prime} \in V$.

Let $r_{1}, r_{2}$ be real numbers, $\infty \geqq r_{2}>r_{1} \geqq 0$. Denote by

$$
f\left(r_{1}, r_{2} ; a_{V}, b_{V}\right)=\int_{r_{1} \leqq y_{v} \|<r_{2}, v \in V} \prod_{v, v^{\prime} \in V}\left\|y_{v}-y_{v^{\prime}}\right\|^{a\left(v, v^{\prime}\right)} \prod_{v \in V}\left\|y_{v}\right\|^{b(v)} d y_{v} .
$$

\section{Lemma 2。}

$$
f\left(r_{1}, r_{2} ; a_{V}, b_{V}\right)=\sum_{V_{1} \cup V_{2}=V} f\left(r_{1}, \infty ; a_{V_{1}}, b_{V_{1}}\right) f\left(0, r_{2} ; a_{V_{2}}, b_{V_{2}}+a_{V_{2}}^{V_{1}}\right)
$$

where the sum goes over all partitions $\left(V_{1}, V_{2}\right)$ of $V$.

Proof. We prove this lemma by induction with respect to the number of elements of $V$. For $|V|=1$ this formula is checked directly:

$$
\begin{aligned}
\int_{r_{1} \leqq y_{i}<r_{2}}\|y\|^{b} d y & =\int_{r_{1} \leqq y y_{i}<r_{2}}\|y\|^{b} d y+\int_{\|y\|<r_{1}}\|y\|^{b} d y+\int_{r_{1} \leqq}\|y\|^{b} d y \\
& =\int_{y_{1}<r_{2}}\|y\|^{b} d y+\int_{r_{1} \leqq}\|y\|^{b} d y
\end{aligned}
$$

(we used $\int_{Q_{p}}\|y\|^{b} d y=0$ ).

Note that

$$
f\left(r_{1}, r_{2} ; a_{V}, b_{V}\right)=f\left(0, r_{2} ; a_{V}, b_{V}\right)-\sum_{V_{1} \subseteq V, V_{1} \neq \varnothing} f\left(0, r_{1} ; a_{V_{1}}, b_{V_{1}}\right) f\left(r_{1}, r_{2} ; a_{V_{2}}, b_{V_{2}}+a_{V_{2}}^{V_{1}}\right) \text {. }
$$

Applying the assumption of induction to $f\left(r_{1}, r_{2} ; a_{V_{2}}, b_{V_{2}}+a_{V_{2}}^{V_{1}}\right)$, we have

$$
\begin{aligned}
f\left(r_{1}, r_{2} ; a_{V}, b_{V}\right)= & f\left(0, r_{2} ; a_{V}, b_{V}\right)-\sum_{V_{1} \subseteq V, V_{1} \neq \varnothing}\left(f\left(0, r_{1} ; a_{V_{1}}, b_{V_{1}}\right)\right. \\
& \left.\cdot \sum_{V_{2} \subseteq V V_{1}} f\left(r_{1}, \infty ; a_{V_{2}}, b_{V_{2}}+a_{V_{2}}^{V_{1}}\right) f\left(0, r_{2} ; a_{V_{3}}, b_{V_{3}}+a_{V_{3}}^{V_{1}}+a_{V_{3}}^{V_{2}}\right)\right),
\end{aligned}
$$

where $V_{3}=V \backslash\left(V_{1} \cup V_{2}\right)$. By denoting $V_{4}=V_{1} \cup V_{2}$ and changing the order of the summation, we get

But

$$
\begin{aligned}
f\left(r_{1}, r_{2} ; a_{V}, b_{V}\right)= & f\left(0, r_{2} ; a_{V}, b_{V}\right)-\sum_{V_{4} \subseteq V, V_{4} \neq \varnothing}\left\{\left(\sum_{V_{1} \subseteq V_{4}, V_{1} \neq \varnothing} f\left(0, r_{1} ; a_{V_{1}}, b_{V_{1}}\right)\right.\right. \\
& \left.\left.\cdot f\left(r_{1}, \infty ; a_{V_{2}}, b_{V_{2}}+a_{V_{2}}^{V_{1}}\right) f\left(0, r_{2} ; a_{V_{3}}, b_{V_{3}}+a_{V_{3}}^{V_{4}}\right)\right)\right\} .
\end{aligned}
$$

$$
\sum_{V_{1} \subseteq V_{4}} f\left(0, r_{1} ; a_{V_{1}}, b_{V_{1}}\right) f\left(r_{1}, \infty ; a_{V_{2}}, b_{V_{2}}+a_{V_{2}}^{V_{1}}\right)=f\left(0, \infty ; a_{V_{4}}, b_{V_{4}}\right)=0,
$$

and hence

$$
\sum_{V_{1} \subseteq V_{4}, V_{1} \neq \varnothing} f\left(0, r_{1} ; a_{V_{1}}, b_{V_{1}}\right) f\left(r_{1}, \infty ; a_{V_{2}}, b_{V_{2}}+a_{V_{2}}^{V_{1}}\right)=-f\left(r_{1}, \infty ; a_{V_{4}}, b_{V_{4}}\right),
$$

where the summation is over $V_{1} \neq \varnothing, V_{1} \subset V_{4}$. Therefore,

$f\left(r_{1}, r_{2} ; a_{V}, b_{V}\right)=f\left(r_{1}, r_{2} ; a_{V}, b_{V}\right)+\sum_{V_{2} \subseteq V_{V_{4}} \neq \varnothing} f\left(r_{1}, \infty ; a_{V_{4}}, b_{V_{4}}\right) f\left(0, r_{2} ; a_{V_{3}}, b_{V_{3}}+a_{V_{3}}^{V_{4}}\right)$,

$V_{3}=V \backslash V_{4}$. Lemma 2 is proved. 
Theorem 1. Let $A_{x}=A$. Then

$$
F(x)=\sum_{I(A)} \prod_{V \in A^{\prime}} C(V, I) \max _{\substack{v \in V \\ v^{\prime} \in V}}\left\|x_{v}-x_{v^{\prime}}\right\|^{\lambda(V, I)}
$$

where

$$
C(V, I)=\int \prod_{v^{\prime}, v \in I(V)}\left\|y_{v}-y_{v^{\prime}}\right\|^{a\left(v, v^{\prime}\right)} \prod_{v \in I(V)}\left(\prod_{V^{\prime} \in K(V)}\left\|y_{v}-\alpha_{V^{\prime}}\right\|^{a\left(v, V^{\prime}(I)\right)} d y_{v}\right),
$$

$\left\{\alpha_{V^{\prime}}, V^{\prime} \in K(V)\right\}$ is an arbitrary set of $p$-adic numbers, such that $\left\|\alpha_{V^{\prime}}-\alpha_{V^{\prime \prime}}\right\|=1$ if $V^{\prime} \neq V^{\prime \prime}$ (for example, $\left.\alpha=\left\{\alpha_{V^{\prime}} ; V^{\prime} \in K(V)\right\}=\{0,1, \ldots,|K(V)|-1\}\right)$. $C(V, I)$ do not depend on the choice of $\alpha$. For the other notations see Sect. 1.

Proof. Let $A_{x}=A$ and $\varphi: A \rightarrow V_{\text {ext }}$ is any function such that $\varphi(V) \in V, V \in A$. Let $T(A)=\{T(V), V \in A\}$ is a partition of $V_{\text {int }}$, indexed by elements of $A$. Denote by

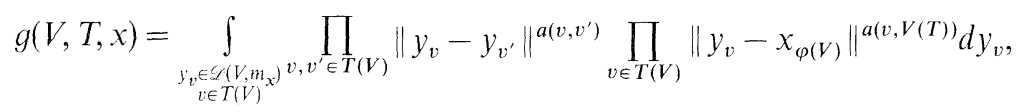

where $V(T)=\left(\bigcup_{V^{\prime} \subset V} T\left(V^{\prime}\right)\right) U V$.

From Lemma 1 it is easy to derive that

$$
F(x)=\sum_{T(A)} \prod_{V \in A^{\prime}} g(V, T, x) m_{x}(V)^{s(V, T)},
$$

where the sum goes over all partitions of $V_{\text {int }}$, indexed by elements of $A$,

$$
s(V, T)=\sum_{\substack{V^{\prime} V^{\prime \prime} \in K(V) \\ V^{\prime} \neq V^{\prime \prime}}} a\left(V^{\prime}(T) \cup T\left(V^{\prime}\right), V^{\prime \prime}(T) \cup T\left(V^{\prime \prime}\right)\right)
$$

According to Lemma 2

$$
g(V, T, x)=\sum_{T_{1}(V) \cup T_{T_{2}(V)}=T(V)} f\left(m(V), \infty ; a_{T_{1}(V)}, a_{T_{1}(V)}^{V(T)}\right) f\left(0, m\left(\tau(V) ; a_{T_{2}(V)}, a_{T_{2}(V)}^{V(T)+T_{1}(V)}\right),\right.
$$

the sum goes over all partitions $\left(T_{1}(V), T_{2}(V)\right)$ of $T(V)$.

As $f\left(0, \infty ; a_{V}, b_{V}\right)=0$ for any $a_{V}, b_{V}$, we may write

$$
\begin{aligned}
F(x)= & \sum_{I(A)} \prod_{V \in A^{\prime}}\left(\sum_{\substack{\left(U T_{2}\left(V^{\prime}\right)\right) U T_{1}(V)=I(V) \\
V^{\prime} \in K(V)}} f\left(m(V), \infty ; a_{T_{1}(V)}, a_{T_{1}(V)}^{V(I) T_{1}(V)}\right)\right. \\
& \left.\cdot \prod_{V^{\prime} \in K(V)} f\left(0, m(V) ; a_{T_{2}(V)}, a_{T_{2}(V)}^{V^{\prime}(I)}\right)\right) m_{x}(V)^{s(V, I)}
\end{aligned}
$$

where the sum goes over all partitions of $V_{\text {int }}$, indexed by elements of $A^{\prime}$, the internal sum taken over all partitions of

$$
\begin{aligned}
I(V) & =\left(\bigcup_{V^{\prime} \in K(V)} T_{2}\left(V^{\prime}\right)\right) \cup T_{1}(V), \quad V(I)=\left(\bigcup_{V^{\prime} \subseteq V} I\left(V^{\prime}\right)\right) \cup V, \\
s(V, I) & =\sum_{\substack{V^{\prime}, V^{\prime \prime} \in K(V) \\
V^{\prime} \neq V^{\prime \prime}}} a\left(V^{\prime}(I), V^{\prime \prime}(V)\right) .
\end{aligned}
$$


As

$\left(\bigcup_{V^{\prime} \in K(V)}\left\{y \in Q_{p}:\left\|y-x_{\varphi\left(V^{\prime}\right)}\right\|<m(V)\right\}\right) \cup\left\{y \in Q_{p}:\left\|y-x_{\varphi(V)}\right\| \geqq m(V)\right\}=Q_{p}$,

we get

$$
\begin{aligned}
F(x)= & \sum_{I(A)} \prod_{V \in A^{\prime}} m_{x}(V)^{s(V, I)} \\
& \cdot\left(\int \prod_{v, v^{\prime} \in I(V)}\left\|y_{v}-y_{v^{\prime}}\right\|^{a\left(v, v^{\prime}\right)} \prod_{v \in I(V)}\left(\prod_{V^{\prime} \in K_{(V)}}\left\|y_{v}-x_{\varphi\left(V^{\prime}\right)}\right\|^{a(v, V(I))}\right) d y_{v}\right) .
\end{aligned}
$$

Performing a change of variables,

$$
\begin{aligned}
& y_{v}=y_{v} p^{-n}, \quad v \in I(V), \\
& \alpha_{V}=x_{\varphi\left(V^{\prime}\right)} p^{-n}, \quad V^{\prime} \in K(V),
\end{aligned}
$$

where $\left\|p^{n}\right\|=m_{x}(V)$, we obtain

$$
\begin{gathered}
F(x)=\sum_{I(A)} \prod_{V \in A^{\prime}} C(V, I, \alpha) m_{x}(V)^{\lambda(V, I)}, \\
C(V, I, \alpha)=\int \prod_{v, v^{\prime} \in I(V)}\left\|y_{v}-y_{v^{\prime}}\right\|^{a\left(v, v^{\prime}\right)} \prod_{v \in I(V)}\left(\prod_{V^{\prime} \in K(V)}\left\|y_{v}-\alpha_{V^{\prime}}\right\|^{a\left(v, V^{\prime}(I)\right)}\right) d y, \\
\lambda(V, I)=a(V(I))-\sum_{V^{\prime} \in K(V)} a\left(V^{\prime}(I)\right)+|I(V)| .
\end{gathered}
$$

Note that $\left\|\alpha_{V^{\prime}}-\alpha_{V^{\prime \prime}}\right\|=1$ for each pair $V^{\prime}, V^{\prime \prime} \in K(V) V^{\prime} \neq V^{\prime \prime}$. In the next section we shall see that $C(V, I, \alpha)$ does not depend on the choice of $\alpha$.

Note also that $C(V, I)$ is a Feynman amplitude of the contracted graph

$$
\left.G_{V}\right|_{\left\{G_{V^{\prime}}, V^{\prime} \in K(V)\right\}} \cdot
$$

Here $G_{V}$ is a graph with the set of vertices $V(I)$. To each external vertice $\left\{V^{\prime}\right\}$ of this contracted graph we must assign an external variable $\alpha_{V^{\prime}}$.

$\lambda(V, I)$ also has a simple geometrical description.

\section{The Calculation of Coefficients $C(V, I)$ and String Amplitudes in the Koba-Nielsen Form}

Let us consider an integral

$$
F_{1}=\int \prod_{v, v^{\prime} \in V}\left\|y_{v}-y_{v^{\prime}}\right\|^{a(v, v)} \prod_{v \in V}\left(\prod_{i=0}^{k}\left\|y_{v}-\alpha_{i}\right\|^{b_{i}(v)}\right) d y_{v},
$$

where $1 \leqq k \leqq p-1,\left\|\alpha_{i}-\alpha_{j}\right\|=1$ if $i \neq j$. This integral is a generalization of Koba-Nielsen amplitude (some examples of this amplitude were calculated in $[10,11])$.

We introduce the next partition of $Q_{p}$ :

$$
\begin{aligned}
& \mathscr{D}_{i}=\left\{y \in Q_{p}:\left\|y-\alpha_{i}\right\|<1\right\}, \quad i=0,1, \ldots, k, \\
& \mathscr{D}_{j}=\left\{y \in Q_{p}:\left\|y-\alpha_{0}\right\|=1,\left(y-\alpha_{0}\right) \bmod p=j\right\}, \quad j \in J .
\end{aligned}
$$


where $J=\{0,1, \ldots, p-1\} \backslash\left\{\left(\alpha_{i}-\alpha_{0}\right) \bmod p, i=0, \ldots, k\right\}$. As $|J|=p-k-1$, it is convenient to renumerate the family $\mathscr{D}_{j}, j \in J$ by $i=k+1, \ldots, p-1$. Finally,

$$
\mathscr{D}_{p}=\left\{y \in Q_{p}:\left\|y-\alpha_{0}\right\|>1\right\} .
$$

As above, it is easy to show that $\left\{\mathscr{D}_{i}, i=0, \ldots, p\right\}$ is a partition of $Q_{p}$. Therefore

$$
\begin{aligned}
& F_{1}=\sum_{\bigcup_{i=0}^{p} V_{i}=V^{i}} \prod_{i=0}^{k} \int_{\substack{v_{v} \in g_{i} \\
v \in V_{i}}} \prod_{v, v^{\prime} \in V_{i}}\left\|y_{v}-y_{v^{\prime}}\right\|^{a\left(v, v^{\prime}\right)} \prod_{v \in V_{i}}\left\|y_{v}-x_{i}\right\|^{b_{i}(v)} d y_{v} \\
& \cdot \prod_{j=k+1}^{p-1} \int_{\substack{y_{v} \in \mathscr{G}_{j} \\
v \in V_{j}}} \prod_{v, v^{\prime} \in V_{j}}\left\|y_{v}-y_{v^{\prime}}\right\|^{a\left(v, v^{\prime}\right)} \prod_{v \in V_{j}} d y_{v} \\
& \cdot \int_{\substack{y_{v} \in \mathscr{V}_{p} \\
v \in V_{p}}} \prod_{\substack{v, v^{\prime} \in V_{i} \\
v^{\prime}}}\left\|y_{v}-y_{v^{\prime}}\right\|^{a\left(v, v^{\prime}\right)} \prod_{v \in V_{i}}\left\|y_{v}-\alpha_{0}\right\|^{\sum_{i=0}^{k} b_{i}(v)+a\left(v, V \backslash V_{p}\right)} d y_{v} .
\end{aligned}
$$

But for $i=0, \ldots, k$,

$$
\begin{aligned}
& \int_{\substack{y_{v} \in \mathscr{G}_{i} \\
v \in V_{i}}} \prod_{v, v^{\prime} \in V_{i}}\left\|y_{v}-y_{v^{\prime}}\right\|^{a\left(v, v^{\prime}\right)} \prod_{v \in V_{i}}\left\|y_{v}-\alpha_{i}\right\|^{b_{i}(v)} d y_{v} \\
& \quad=\int_{\substack{y_{v}<1 \\
v \in V_{i}}} \prod_{v, v^{\prime} \in V_{i}}\left\|y_{v}-y_{v^{\prime}}\right\|\left\|^{a\left(v, v^{\prime}\right)} \prod_{v \in V_{i}}\right\| y_{v}-\alpha_{i} \|^{b_{i}(v)} d y_{r},
\end{aligned}
$$

for $i=k+1, \ldots, p-1$

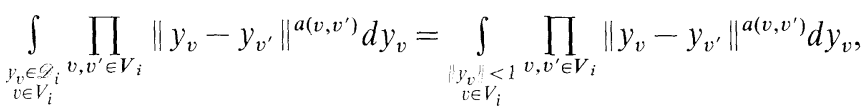

and

$$
\begin{aligned}
& \int_{\substack{y_{v} \in \mathscr{O}_{p}, p \\
v \in V_{p}^{p}}} \prod_{\substack{v^{\prime} \in V_{p}\\
}}\left\|y_{v}-y_{v^{\prime}}\right\|^{a\left(v, v^{\prime}\right)} \prod_{v \in V_{p}}\left\|y_{v}-\alpha_{0}\right\|^{\sum_{i=0}^{k} b_{i}(v)+a\left(v, V \backslash V_{p}\right)} d y_{v} \\
& =\int_{\substack{y_{v}<1 \\
v \in V_{p}}} \sum_{v, v^{\prime} \in V_{p}}\left\|y_{v}-y_{v^{\prime}}\right\|^{a\left(v, v^{\prime}\right)} \prod_{v \in V_{p}}\left\|y_{v}\right\|^{\left.\sum_{i=0}^{k} b_{i}(v)+a(v, V)+2\right)} d y_{v} .
\end{aligned}
$$

In the last reduction we used the change of variables $y_{v} \rightarrow\left(1 / y_{v}-\alpha_{0}\right), v \in V_{p}$. Therefore, we may rewrite

where

$$
F_{1}=\sum_{\cup_{i=0}^{n} V_{i}=V^{i}} \prod_{i=0}^{p} g\left(a_{V_{i}}, b_{i}\right)
$$

$$
\begin{aligned}
g\left(a_{V_{i}}, b_{i}\right) & =\int_{\substack{\mid y_{v} \|_{v \in V_{i}} \\
v}} \prod_{v, v^{\prime} \in V_{i}}\left\|y_{v}-y_{v^{\prime}}\right\|^{a\left(v, v^{\prime}\right)} \prod_{v \in V_{i}}\left\|y_{v}\right\|^{b_{i}(v)} d y_{v}, \\
b_{i}(v) & =0, \quad v \in V_{i}, \quad i=k+1, \ldots, p-1, \\
b_{p}(v) & \left.=-\left(\sum_{i=0}^{k} b_{i}(v)+a(v, V)+2\right)\right), \quad v \in V_{p} .
\end{aligned}
$$


So, we must calculate the integral of the type

$$
F_{2}\left(a_{V}\right)=\int_{\substack{\left|y_{v}\right|<1 \\ v \in V \backslash\left\{v_{0}\right\}}} \prod_{v, v^{\prime} \in V}\left\|y_{v}-y_{v^{\prime}}\right\|^{a\left(v, v^{\prime}\right)} \prod_{v \in V \backslash\left\{v_{0}\right\}} d y_{v},
$$

where $v_{0}$ is a fixed element of $V, y_{v_{0}}=0, a\left(v, v^{\prime}\right) \in \mathbb{C}$ for every pair $\left(v, v^{\prime}\right)$ (we identify each pair $\left(v, v^{\prime}\right)$ with $\left.\left(v^{\prime}, v\right)\right), a(v, v)=0$ for every $v \in V$ (in our case $V \backslash\left\{v_{0}\right\}=V_{i}$, $\left.a\left(v, v_{0}\right)=b_{i}(v), i=0,1, \ldots, p\right)$.

Lemma 3. Let $(A, m)$ be an indexed hierarchy on $V$, and

$$
\chi_{A, m}\left(y_{v} ; v \in V \backslash\left\{v_{0}\right\}\right)= \begin{cases}1, & \text { if }\left(A_{y}, m_{y}\right)=(A, m) \\ 0, & \text { otherwise }\end{cases}
$$

where $y=\left\{y_{v}, v \in V\right\}, y_{v_{0}}=0$. Then

$$
\int y_{A, m}\left(y_{i} ; v \in V \backslash\left\{v_{0}\right\}\right) \prod_{v \in V\left\{v_{0} !\right.} d y_{v}=\prod_{V^{\prime} \in A^{\prime}}\left(\frac{m\left(V^{\prime}\right)}{p}\right)^{\left|K\left(V^{\prime}\right)\right|-1} \frac{(p-1) !}{(p-|K(V)|) !} .
$$

Proof of Lemma 3. Let $T$ be a tree with the set of vertices $V$ and the set of lines $L=\left\{l=\left(\varphi\left(V^{\prime}\right), \varphi\left(V^{\prime \prime}\right)\right), \varphi\left(V^{\prime}\right) \neq \varphi\left(V^{\prime \prime}\right) \mid V^{\prime} \in A, \quad V^{\prime \prime} \in K\left(V^{\prime}\right)\right\}$. Here $\varphi: A \rightarrow V$ is any function such that $\varphi\left(V^{\prime}\right) \in V^{\prime}$ for every $V^{\prime} \in A, \varphi(V)=v_{0}$. To each line $l=\left(\varphi\left(V^{\prime}\right)\right.$, $\left.\varphi\left(V^{\prime \prime}\right)\right)$ we assign a variable $S_{l}=y_{\varphi\left(V^{\prime}\right)}-y_{\varphi\left(V^{\prime \prime}\right)}$. Then for every $y_{v}=S_{l_{1}}+S_{l_{2}}+$ $\cdots+S_{l_{j}}$, where $\left\{l_{1}, \ldots, l_{j}\right\}$ is a path joining $v$ with $v_{0}$. It is clear that jacobian of this change of variables is equal to 1 . And moreover, $\left(A_{y}, m_{y}\right)=(A, m)$ if and only if

1) $\left\|S_{l}\right\|=m\left(V^{\prime}\right)=p^{n\left(V^{\prime}\right)}$ for every $l=\left(\varphi\left(V^{\prime}\right), \varphi\left(V^{\prime \prime}\right)\right), V^{\prime} \in A^{\prime}, V^{\prime \prime} \in K\left(V^{\prime}\right)$.

2) $S_{l_{1}} p^{n\left(V^{\prime}\right)} \bmod p \neq S_{l_{2}} p^{n\left(V^{\prime}\right)} \bmod p$ for each pair $l_{1}=\left(\varphi\left(V^{\prime}\right), \varphi\left(V_{1}\right)\right)$,

$l_{2}=\left(\varphi\left(V^{\prime}\right), \varphi\left(V_{2}\right)\right), V^{\prime} \in A^{\prime}, V_{1} \in K\left(V^{\prime}\right), V_{2} \in K\left(V^{\prime}\right), V_{1} \neq V_{2}$.

Using

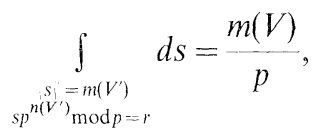

$r=1,2, \ldots, p-1$, we obtain the formula (4.15).

Theorem 2. Let $a\left(V^{\prime}\right)+\left|V^{\prime}\right|-1>0$ for every $V^{\prime} \subseteq V,\left|V^{\prime}\right|>1$. Then

$$
F_{2}\left(a_{V}\right)=p^{-|V|+1} \sum_{A} \prod_{V^{\prime} \in A^{\prime}} \frac{1}{p^{a\left(V^{\prime}\right)+\left\{V^{\prime} \mid-1\right.}-1} \cdot \frac{(p-1) !}{\left(p-\left|K\left(V^{\prime}\right)\right|\right) !},
$$

the sum goes over all hierarchies $A$ on $V$.

Proof.

$$
g\left(a_{V}\right)=\sum_{(A, m): m(V)<1} \int \prod_{v, v^{\prime} \in V}\left\|y_{v}-y_{v^{\prime}}\right\|^{a\left(v, v^{\prime}\right)} \chi_{A, m}\left(y_{v} ; v \in V \backslash\left\{v_{0}\right\}\right) \prod_{v \in V \backslash\left\{v_{0}\right\}} d y_{v}
$$

where the sum taken over all indexed hierarchies on $V$, such that $m(V)<1$. Note that this sum is equal to the double sum

$$
\sum_{A} \sum_{m: n(V)<0} \int \prod_{v, v^{\prime} \in V}\left\|y_{v}-y_{v^{\prime}}\right\|^{a\left(v, v^{\prime}\right)} \chi_{A, m}\left(y_{v} ; v \in V \backslash\left\{v_{0}\right\}\right) \prod_{v \in V \backslash\left\{v_{0}\right\}} d y_{v}
$$


where the external sum goes over all hierarchies on $V$ and the internal sum goes over all set of integer numbers $\left\{n\left(V^{\prime}\right), V^{\prime} \in A^{\prime}\right\}, n\left(V^{\prime}\right) \in Z, m\left(V^{\prime}\right)=p^{n\left(V^{\prime}\right)}, n\left(V^{\prime}\right)<n\left(\tau\left(V^{\prime}\right)\right)$ for every $V^{\prime} \in A^{\prime}$ and $n(V)<0$.

Let

$$
a^{\prime}\left(V^{\prime}\right)=a\left(V^{\prime}\right)-\sum_{V^{\prime \prime} \in K\left(V^{\prime}\right)} a\left(V^{\prime \prime}\right), \quad V^{\prime} \in A^{\prime}
$$

Obviously, that

$$
\prod_{v, v^{\prime} \in V}\left\|y_{v}-y_{v^{\prime}}\right\|^{a\left(v, v^{\prime}\right)} \chi_{A, m}\left(y_{v} ; v \in V \backslash\left\{v_{0}\right\}\right)=\prod_{V^{\prime} \in A^{\prime}} m\left(V^{\prime}\right)^{a^{\prime}\left(V^{\prime}\right)} \chi_{A, m}\left(y_{v} ; v \in V \backslash\left\{v_{0}\right\}\right) .
$$

Note that if $\beta>0$, then

$$
\sum_{n<m} p^{n \beta}=\frac{p^{m \beta}}{p^{\beta-1}} .
$$

Using (4.21) and Lemma 3, we obtain

$$
\begin{gathered}
\sum_{A} \sum_{n: m\left(V^{\prime}\right)<0} \sum_{V^{\prime} \in A^{\prime}} p^{n\left(V^{\prime}\right)\left(\left(^{\prime}\left(V^{\prime}\right)+\left|K\left(V^{\prime}\right)\right|+1\right)\right.} p^{-\left|K\left(V^{\prime}\right)\right|+1} \cdot \frac{(p-1) !}{\left(p-\left|K\left(V^{\prime}\right)\right|\right) !} \\
==\sum_{A} \prod_{V^{\prime} \in A^{\prime}} p^{-\left|K\left(V^{\prime}\right)\right|+1} \cdot \frac{(p-1) !}{\left(p-\left|K\left(V^{\prime}\right)\right|\right) ! p^{\beta\left(V^{\prime}\right)}-1} .
\end{gathered}
$$

Here we used that

$$
\beta\left(V^{\prime}\right)=\sum_{V^{\prime \prime} \subseteq V^{\prime}, V^{\prime \prime} \in A^{\prime}}\left(a^{\prime}\left(V^{\prime \prime}\right)+\left|K\left(V^{\prime \prime}\right)\right|-1\right)>0 .
$$

As

$$
\begin{gathered}
\sum_{V^{\prime \prime} \in V^{\prime}, V^{\prime} \in A^{\prime}} a^{\prime}\left(V^{\prime \prime}\right)=a\left(V^{\prime}\right), \\
\sum_{V^{\prime \prime} \leqq V^{\prime}, V^{\prime \prime} \in A^{\prime}}\left(\left|K\left(V^{\prime \prime} \mid-1\right)=\right| V^{\prime} \mid-1,\right.
\end{gathered}
$$

we have

$$
\beta\left(V^{\prime}\right)=a\left(V^{\prime}\right)+\left|V^{\prime}\right|-1>0, \quad V^{\prime} \subseteq V
$$

by the assumption of the theorem. Finally we get

$$
F_{2}\left(a_{V}\right)=p^{-\mid V^{\prime}+1} \sum_{A} \prod_{V^{\prime} \in A^{\prime}} \frac{1}{p^{a\left(V^{\prime}\right)+\left|V^{\prime}\right|-1}-1} \cdot \frac{(p-1) !}{\left(p-\left|K\left(V^{\prime}\right)\right|\right) !} .
$$

The theorem is proved.

This last formula defines also an analytic continuation of $F_{2}\left(a_{V}\right)$ from the domian (4.29) to the whole complex plane as a meromorphic function of $a\left(v, v^{\prime}\right)$, $v, v^{\prime} \in V$.

\section{References}

i. Lerner, E. X., Missarov, M. D.: The scalar models of $p$-adic quantum field theory and hierarchical model of Dyson, submitied io "Theoretical and Mathematical physics" 
2. Bleher, P. M., Sinai, Y. G.: Commun. Math. Phys. 45247 (1975)

3. Collet, P., Eckmann, J.-P.: A renormalization group analysis of the hierarchical model in statistical mechanics. Lecture Notes in Physics vol. 74, pp. 1-199. Berlin, Heidelberg, New York: Springer 1978

4. Bleher, P. M.: Commun. Math. Phys. 84, 557 (1982)

5. Gawedzki, K., Kupiainen, A.: J. Stat. Phys. 29, 683 (1982)

6. Volovich, I. V.: Number theory as the ultimate physical theory, preprint CERN-TH.4781/87

7. Volovich, I. V.: Class. Quant. Grav. 4, L83 (1987)

8. Grossman, B.: Phys. Lett. B, 197, 101 (1987)

9. Freund, P. O. G., Olson, M.: Non-Archimedian Strings. Chicago, IL: University of Chicago, preprint EFI-87-54

10. Freund, P. G. O., Witten, E.: Adelic String Amplitudes, Institute for Advanced Studies, preprint IASSNS-HEP-87/42

11. Gervais, J. L.: p-adic Analyticity and Virasoro Algebras for Conformal Theories in more than two dimensions, Ecole Normale, Preprint LPTENS 87/32 (1987)

12. Brekke, L., Freund, P. G. O., Olson, M., Witten, E.: Non-Archimedian String Dynamics, University of Chicago, Preprint EFI-87-101

13. Marinari, E., Parisi, G.: On the p-adic Five Point Function, Preprint ROM2F-87/38 (1987)

14. Rammal, R., Toulouse, G., Virasoro, M. A.: Rev. Mod. Phys. 58, 699 (1986)

15. Gel'fand, I. M., Graev, M. I., Pyatetskii-Shapiro, I. I.: Representation theory and Automorphic functions. London: Saunders 1966

16. Bleher, P. M., Missarov, M. D.: Commun. Math. Phys. 74, 255 (1980)

Communicated by K. Gawedzki

Received June 21, 1988; in revised form August 3, 1988 